\title{
Criminologie
}

\section{Guide de lecture sur le vol à main armée : si le coeur vous en dit !}

\section{André Normandeau et Maurice Cusson}

Volume 18, numéro 2, 1985

Le vol à main armée à Montréal

URI : https://id.erudit.org/iderudit/017221ar

DOI : https://doi.org/10.7202/017221ar

Aller au sommaire du numéro

Éditeur(s)

Les Presses de l'Université de Montréal

ISSN

0316-0041 (imprimé)

1492-1367 (numérique)

Découvrir la revue

Citer ce document

Normandeau, A. \& Cusson, M. (1985). Guide de lecture sur le vol à main armée : si le coeur vous en dit ! Criminologie, 18(2), 147-154.

https://doi.org/10.7202/017221ar d'utilisation que vous pouvez consulter en ligne.

https://apropos.erudit.org/fr/usagers/politique-dutilisation/ 
GUIDE DE LECTURE SUR LE VOL À MAIN ARMÉE :

SI LE COEUR VOUS EN DIT !

André Normandeau*

Maurice Cusson**

La littérature scientifique et populaire sur le vol à main armée est abondante, autant au niveau des livres que des articles. Il va donc de soi que ce guide est une «sélection». Dans la première partie, consacrée aux travaux réalisés au Québec, nous avons sélectionné la littérature scientifique au chapitre des rapports de recherche, des livres et des articles depuis 1960. Dans la seconde partie, consacrée aux travaux à l'étranger, nous avons privilégié les livres scientifiques seulement.

\section{LE VOL À MAIN ARMÉE AU QUÉBEC : 1960-1985}

a. LES PIONNIERS : $1966-1980$

$\neg$ TARDIF, G. (1966), les Crimes de violence, Thèse de maîtrise, Département de criminologie, Université de Montréal. Rúsumé-synthèse sous forme d'article in Actes du Ve Colloque de recherche de la Société de criminologie du Québec, Montréal, Centre de psychologie et de pédagogie, pp. 53-72.

- PRONOVOST, L., D. SZADO et G. TARDIF (1968), «Le banditisme à Montréal : caractéristique des vols bancaires à Montreal», in Actes du 11e Congrès de la Société de criminologie du Québec, Montréal, Editions Beauchemin, pp. 139-152.

- COMMISSION (PRÉVOST) D'ENQUÊTE SUR L'ADMINISTRATION DE LA JUSTICE AU QUÉBEC (1970), le Crime au Québec : les sommets de la criminalité québécoise (vol. 3, t. II), Québec, Editeur officiel. Lire en particulier la seconde partie intitulée : «Le vol à main armé», pp. 161-225.

- COMMISSION DE POLICE DU QUÉBEC (1976), Étude sur les vols à main armée dans les institutions bancaires au Québec, Québec, ministère de la Justice, 8 vol.

- ASSOCIATION DES BANQUIERS CANADIENS ET SERVICE DE POLICE DE LA COMMUNAUTÉ URBAINE DE MONTRÉAL (1977), Étude sur les vols de banque de la Communauté urbaine de Montréal. En version anglaise également.

- BARIL, M. (1977), l'Image de la violence au Québec, Montréal, Centre international de criminologie comparée, Université de Montréal, $260 \mathrm{p}$.

- BARIL, M., H. GRENIER, D. LEMAIRE et H. MANSEAU (1978), les Petits Commerçants victimes de vol à main armée : en quête dé

* Directeur, Centre international de criminologie comparée, Université de Montréal.

** Professeur titulaire à l'École de criminologie et chercheur associé au Centre international de criminologie comparée, Université de Montréal. 
justice, Montréal, Centre international de criminologie comparée, Université de Montréal, $185 \mathrm{p}$.

- BARIL, M., L. HUOT et J. GIROUX (1978), le Citoyen victime de vol qualifié : sa place dans le processus judiciaire, Montréal, Centre international de criminologie comparée, Université de Montréal, 337 p.

- HUOT, L., J. GIROUX (1979), «Le citoyen et la justice», Criminologie, 12,1, 43-56.

- MANSEAU, H., H. GRENIER (1979), «Les petits commerçants victimes de vol a main armée : en quête de justice», Criminologie, 12. 1, 57-65.

- BARIL, M. (1979), «Violence et répression : mythe ou réalité», Criminologie, 12, 1, 66-77.

- BARIL, M. (1980), «Ils n'ont plus la liberté : réactions à la victimisation et ses conséquences», Criminologie, 13, 1, 94-103.

\section{b. LE RAPPORT LAPLANTE ET SES ANNEXES} DE RECHERCHE : 1980

- LAPLANTE, L. (président), A. NORMANDEAU (secrétaire) (1980), le Vol à main armée au Québec, Québec, ministère de la Justice, $245 \mathrm{p}$.

- LAPLANTE, L. (président), A. NORMANDEAU (secrétaire) (1980), le Vol à main armée au Québec. Version populaire en format tabloïd publiée par le journal la Flâte de la Fraternité des policiers de la Communauté urbaine de Montréal, $76 \mathrm{p}$.

- LAPLANTE, L. (président), A. NORMANDEAU (secrétaire) (1980), Annexes de recherche sur le vol à main armée au Québec (polycopiées), Québec, ministère de la Justice, Montréal, Centre international de criminologie comparée, Université de Montréal.

- GROUPE DE TRAVAIL SUR LE VOL A MAIN ARMÉE, G.T.V.O.L., no 1, (B. Marceau), le Vol d̀ main armée à Montréal, $90 \mathrm{p}$.

- G.T.V.O.L. n० 2 (B. Marceau), Étude sur les sentences prononcées en matière de vol à main armée dans le district de Montréal, $27 \mathrm{p}$.

- G.T.V.O.L. n० 3 (H. Manseau), les Vols à main armée tels que vus par des victimes, $73 \mathrm{p}$.

- G.T.V.O.L. no 4 (M. Desrochers), les Victimes de vols à main armée, $106 \mathrm{p}$.

- G.T.V.O.L. no 5 (M.J. Lavigueur, S. Bruneau), le Vol à main armée chez les juvéniles à Montréal, 97 p.

- G.T.V.O.L. n० 6 (R. Lanciault), la Carrière du voleur à main armée au Quebec, 53 p.

- G.T.V.O.L. no7 (N. Henry, J. Cliche), le Voleur à main armée au Québec : le phénomène, $124 \mathrm{p}$.

c. LE RAPPORT LAPLANTE : ARTICLES DE 1980 A 1983

\section{ARTICLES DE VULGARISATION}

- NORMANDEAU, A. (1980), «Le vol à main armée au Québec», le Devoir, 18 octobre 1980 , p. 11. 
- NORMANDEAU, A. (1980), «Le vol à main armée vu au bout du canon», le Soleil, 31 octobre 1980, p. 7.

- NORMANDEAU, A. (1980), «Pour une ligue des droits et libertés des victimes», Actes, Congrès de la Société de Criminologie du Québec, 379-386.

- NORMANDEAU, A. (1981), «Pour une ligue des droits et libertés des victimes», la Flâte, 14, 2, 42-43, Montréal.

- NORMANDEAU, A. (1981), \&Les droits et libertés des victimes», Déviance et société, 5, 3, 283-287.

- NORMANDEAU, A. (1981), «Les droits et libertes des victimes», Revue internationale de police criminelle, 36, 351, 229-231. En version anglaise, espagnole et arabe, également.

- CUSSON, M., P. LANDREVILLE (1981), «Le vol à main armée au Québec», la Flûte, 14, 2, 58-59.

- NORMANDEAU, A. (1982), «Toward a League for Victims' Rights and Liberties», Canadian Journal of Criminology, 24, 1, 87-91.

- LANCIAULT, R., A. NORMANDEAU (1983), «La carrière du voleur à main armée», Sûreté, 13, 7, 10-17.

\section{ARTICLES SCIENTIFIQUES}

- NORMANDEAU, A. (1980), «Le phénomène du vol à main armée en Amérique», Revue internationale de criminologie et de police technique, 33, 4, 379-390.

- LAPLANTE, L., B. MARCEAU, A. NORMANDEAU (1980), kLes sentences pour vols à main armée à Montréal», le Forum canadien de criminologie, 3, 1, 57-64.

- BARIL, M., P. LANDREVILLE (1981), «Rapport sur le vol à main armée au Québec», Revue canadienne de criminologie, 23, 2, 217-229.

- BEllemare, R., A. NORMANDEAU (1981), aThe Police and Armed Robbery in Canada», The Police Journal, 54, 4, 390-398.

- BRUNEAU, A., M.J. LAVIGUEUR, A. NORMANDEAU (1981), «Le vol à main armée et les mineurs à Montréal», Apprentissage et socialisation, 4, 2, 101-115.

- LAPLANTE, L., A. NORMANDEAU (1981), «Les facteurs du vol à main armée au Canada», Revue de science criminelle et de droit pénal comparé, 60, 3, 593-600.

- MANSEAU, H., A. NORMANDEAU (1981), «Les victimes du vol a main armée», Revue de droit pénal et de criminologie, 75, 6, 545-560.

- MARCEAU, B., A. NORMANDEAU (1981), «Le vol à main armée à Montréal», Revue canadienne de criminologie, 23, 1, 127-145.

- NORMANDEAU, A. (1981), «Le vol à main armée au Québec», Criminologie, 14, 1, 105-110.

- NORMANDEAU, A. (1981), «Le vol à main armée au Québec et le processus judiciaire», la Revue du Barreau du Québec, 41, 1, 81-98.

- NORMANDEAU, A. (1981), «Le phénomène du vol à main armée en Amérique», Journal du collège canadien de police, 5, 1, 1-13. 
- NORMANDEAU, A. (1981), «Armed Robbery in America», Canadian Police College Journal, 5, 1, 1-12.

- NORMANDEAU, A. (1981), \&Armed Robbery in Montreal and its Victims», Victimology, $10,2,306-317$.

— LANCIAULT, R., A. NORMANDEAU (1983), «La carrière du voleur», Revue canadienne de criminologie, 25, 1, 33-46.

- NORMANDEAU, A., J. PARÉ (1983), «Sentencing and Armed Robbery», Canadian Criminology Forum, 5, 2, 150-153.

d. LE COLLECTIF DE L'UNIVERSITÉ DE MONTRÉAL SUR LE VOL A MAIN ARMÉE : 1980-1985

\section{LE PROJET INITIAL DU COLLECTIF}

- ELIE, D., M. LEBLANC, A. NORMANDEAU (1980), le Vol à main armée : étude compréhensive, Centre international de criminologie comparée, Université de Montréal, 23 p., projet initial (F.C.A.C.).

\section{LES RAPPORTS}

- BELloT, S., D. ELIE, (1983), le Vol à main armée au Québec : description statistique, Centre international de criminologie comparé, Université de Montréal, rapport final no 1, 274 p.

— BELlOT, S., M. DIONNE, P. PINSONNEAULT, M. CUSSON (1984), le Vol d̀ main armée décrit par ses auteurs, Centre international de criminologie comparée, Université de Montréal, rapport final no 2, $153 \mathrm{p}$.

- GAGNON, R., M. LEBLANC (1984), le Vol à main armée dans les systemes de justice, Centre international de criminologie comparée, Université de Montréal, rapport final no 3, $357 \mathrm{p}$.

- BARIL, M., A. MORISSETTE (1985), les Victimes et la prévention du vol à main armée, Centre international de criminologie comparée, Université de Montréal, rapport final no 4 (titre provisoire).

- NORMANDEAU, A., P. PINSONNEAULT (1985), le Vol à main armée à Montréal : les voleurs parlent, les victimes se prononcent..., Centre international de criminologie comparée, Univensité de Montréal, rapport final no 5 .

\section{LES RAPPORTS TECHNIQUES DU COLLECTIF}

- CHARLAND, R. (1981), le Vol à main armée au Québec : inventaire des écrits, Centre international de criminologie comparée, Université de Montréal, rapport technique no 1, $126 \mathrm{p}$.

- NORMANDEAU, A. (1982), le Vol à main armée au Québec : études empiriques préliminaires, Centre international de criminologie comparée, Université de Montréal, rapport technique n० 2, $178 \mathrm{p}$.

- BELLOT, S., R. GAGNON (1983), le Vol à main armée au Québec : bibliographie thématique, Centre international de criminologie comparée, Université de Montréal, rapport technique no 3, $87 \mathrm{p}$. 
- HÉTU, F. (1983), le Vol de banque à Montréal, Centre international de criminologie comparée, Université de Montréal rapport technique no 4 , $231 \mathrm{p}$.

- KAPÉTANAKI, A. (1983), l'Efficacité du contrôle de la police face aux vols et aux vols à main armée, Centre international de criminologie comparée, Université de Montréal, rapport technique no 5, 198 p.

- DIARRA, B. (1983), le Vol à main armée au Québec : législation procédure et «sentencing», Centre international de criminologie comparée, Université de Montréal, rapport technique no 6, $289 \mathrm{p}$.

- BELLOT, S. (1983), Portrait du voleur à main armée occasionnel. Centre international de criminologie comparée, Université de Montréal, rapport technique $n^{\circ} \mathbf{7 , 7 1} \mathrm{p}$.

- PINSONNEAULT, P. (1984), l'Abandon de la carrière criminelle, Centre international de criminologie comparée, Université de Montréal. rapport technique no 8, $198 \mathrm{p}$. Aussi in les Cahiers de l'École de criminologie, rapport no 15 .

- DIONNE, M. (1984), Portraits de voleurs à main armée : les récidivistes et les professionnels, Centre international de criminologie comparée, Université de Montréal, rapport technique no 9, 199 p.

- BELlOT, S., M. LEBLANC (1984), le Vol à main armée parmi l'activité délinquante, Centre international de criminologie comparée, Université de Montréal, rapport technique no 10, 58 p.

- MORISSETTE. A. (1985), Subir un vol à main armée : réactions et conséquences, Centre international de criminologie comparée, Université de Montréal, rapport technique no 11, $264 \mathrm{p}$.

- CANTIN, R. (1985), les Critères de solution des vols à main armée à Québec, Centre international de criminologie comparée, Univensitê de Montréal, rapport technique no 12, $230 \mathrm{p}$.

- POIRIER, D. (à venir), Prévention et vol à main armée, Centre international de criminologie comparée, Université de Montréal, rapport technique no 13 (titre provisoire).

- TRUDEL, L. (à venir), les Cibles des voleurs à main armée, Centre international de criminologie comparée, Université de Montréal, rapport technique no 14 (titre provisoire).

\section{LES COMMUNICATIONS SCIENTIFIQUES DU COLLECTIF}

- BARIL, M. (1984), «Victims of Robbery and Prevention», Conférence au Congrès annuel de l'American Society of Crininology, Cincinnati, Ohio, 7-10 novembre 1984.

- CUSSON, M. (1984), «The Career of Robbers : Getting in and out», Conférence au Congrès annuel de l'American Society of Criminology, Cincinnati, Ohio, 7-10 novembre 1984.

- ELIE, D., A. NORMANDEAU (1985), «Armed Robbery in North America : Current Trends», Conférence au Congrès annuel de l'American Society of Criminology, Cincinnati, Ohio, 7-10 novembre 1984. 
- LEBLANC, M. (1984), «Armed Robbery through the Criminal Justice System», Conférence au Congrès annuel de l'American Society of Criminology, Cincinnati, Ohio, 7-10 novembre 1984.

\section{LES ARTICLES SCIENTIFIQUES DU COLLECTIF}

- ELIE, D. (1981), «La criminalité au Québec», Criminologie, 14, 1, 85-104.

- BARIL, M. (1983), «Une illustration de la peur concrète : le cas des victimes», Criminologie, 16, 1, 31-49.

- ELIE, D., F. HÉTU (1983), «The War Against Hold-ups», Canadian Banker, 10, 5, 40-42.

- GAGNON, R., M. LEBLANC (1983), «L'intervention policière en matière de vol à main armée», Journal du collège canadien de police, 7, 4, 317-330.

- GAGNON, R., M. LEBLANC (1983), «Police Response in Armed Robbery Cases», Canadian Police College Journal, 7, 4, 297-309.

- GAGNON, R., M. LEBLANC (1985), «Une typologie des vols à main armée à Québec et à Montréal», Revue canadienne de criminologie, 27, 1, 31.42.

- ELIE, D. (1985), «En analysant les statistiques des vols de banque à Montréal», Revue internationale de criminologie et de police technique, 38, 1.

- ELIE, D., A. NORMANDEAU, (1985), «Armed Robbery in North America : Current Trends», Canadian Journal of Criminology, 27, 2,

- ELIE, D., A. NORMANDEAU (1985), \&Le vol à main armée», le banquier, 12, 1, 38-41.

- ELIE, D., A. NORMANDEAU (1985), «Armed Robbery», Canadian Banker, 12, 1, 36-39.

- NORMANDEAU, A., J. RICO (1985), «Tendances et configurations de la criminalité au Québec : 1960-1985», in M. LeBlanc et D. Szabo (édit.), Traité de criminologie empirique, Montréal, Les Presses de l'Université de Montréal (sous presse).

6. THÈSE DE DOCTORAT PARTIELLEMENT RELIÉE AU THÈME DU VOL QUALIFIÉ ET DU VOL À MAIN ARMÉE

- BARIL, M. (1983), l'Envers du crime, Thèse de doctorat, École de criminologie, Université de Montréal, $457 \mathrm{p}$.

II. LE VOL À MAIN ARMÉE AU CANADA AUX ÉTATS-UNIS ET AILLEURS : 1960-1985

a. CANADA

- LETKEMANN, P. (1973), Crime as Work, New Jersey, Prentice Hall. 
- BILliNgSLEY, B., C. CANFIELD et B. HANN (1982), Robbery in Canada : an Exploratory Study, Toronto, The Research Group, 129 p.

- DESROCHES, F. (1985), Robbery in Ontario, University of Waterloo, Projet en cours.

\section{b. ÉTATS-UNIS}

\section{L'ÉTUDE DE PHILADELPHIE}

- NORMANDEAU, A. (1968), Trends and Patterns in Crimes of Robbery, Ph.D. dissertation, Philadelphia, University of Pennsylvania, $450 \mathrm{p}$.

- NORMANDEAU, A. (1968), «Patterns in Robbery», Criminologica, 6, 3, 2-15.

- NORMANDEAU, A. (1968), "Notes sur les crimes de violence», Annales internationales de criminologie, 7, 2, 393-402.

- NORMANDEAU, A. (1969), «Trends in Robbery as Reflected by Different Indexes», in T. Sellin et M.E. Wolfgang (édit.), Delinquency : selected studies, New York, Wiley, 150-157.

- NORMANDEAU, A. (1969), «Robbery in Philadelphia and London», British Journal of Criminology, 9, 1, 71-79.

- NORMANDEAU, A. (1972), «Violence and Robbery : a Case Study», Acta Criminologica, 5, 1, 11-106.

\section{AUTRES RECHERCHES SÉLECTIONNÉES}

- BUREAU OF JUSTICE STATISTICS, BULLETIN (1984), Bank Robbery, Washington, D.C. : U.S. Department of Justice.

- CAMP, G. (1968), Nothing to Lose : a Study of Bank Robbery in America, Thèse de doctorat, Department of Sociology, Yale University.

- CONKLIN, J.E. (1972), Robbery and the Criminal Justice System, Philadelphia, Lippincott.

- COOK, P.J. (1983), Robbery in the United States : an Analysis of Recent Trends and Patterns, Washington, D.C., U.S. Department of Justice.

- CURTIS, L. (1974), Criminal Violence, New York, Free Press.

- EISTADTER, T. (1966), Armed Robbery - A Career Study in Perspective, Thèse de doctorat, Department of Sociology, University of California at Berkeley.

- FENNEY, F., A. WEIR (1973), The Prevention and Control of Robbery, Center on Criminal Justice, University of California at Davis, 5 vol.

- HUNT, M. (1972), The Mugging, New York, Atheneum.

- MacDONALD, J. (1975), Armed Robbery, Springfield, Charles Thomas.

- MULTIVILL, D., M. TUMIN (1969), Crimes of Violence, Washington, D.C., U.S. G.P.O. 
- WOLCOTT, G. (1968), A Typology of Armed Robbers, Thèse de maitrise, Department of Sociology, Sacramento State College.

c. BELGIQUE, ANGLETERRE, FRANCE

- BEKAERT, H. et al. (1970), Hold-up en Belgique, Université de Bruxelles.

- McCLINTOCK, F.H., E. GIBSON (1961), Robbery in London, London, MacMillan.

— PEyrefitTe, A. (1977), Réponse à la violence, Paris, Presses Pocket. 